\title{
Comparative Study on Exploring General Mental Health of University Learners
}

\author{
Shahrina Ferdous ${ }^{1}$, Arifa Rahman ${ }^{2}$ \\ ${ }^{1}$ Green University of Bangladesh, Psychosocial Counseling Unit (PSCU), 220/D, Begum Rokeya Sarani, Dhaka-1207, Bangladesh \\ ${ }^{2}$ Green University of Bangladesh, Center of Excellence for Teaching and Learning, 220/D, Begum Rokeya Sarani, Dhaka-1207, Bangladesh
}

\begin{abstract}
The present research was conducted to investigate the mental health status of Bangladesi private and public university learners. Samples of 503 students from different universities were randomly selected. The General Health Questionnaire (GHQ-28) and counseling sessions were used in the mixed method approach. Multi-instrument analysis technique was used.The frequency of somatic problem, anxiety \& sleep problem in severe cases were only $7 \%$ whereas students has less problem related to social functioning (3\%) and depression (2\%) and $78 \%$ students were nonclinical cases and only $12 \%$ students were considered as clinical cases. The mental health of male learners was healthier than females in terms of somatic problem and anxiety \& sleep problem. Conversely, male students had more problems regarding social functioning. Among the students of 20 years to 27 years, maximum were quite sound in terms of depression and have moderate and sound mental health. Afterwards, the tendency of sound mental health was decreasing due to career concern and academic pressure. A little significant differences also exist between public and private university learners' general mental health. Psychological services can play a noteworthy role in maintaining their psychological wellbeing as well as their personal and professional wellbeing.
\end{abstract}

Keywords: Comparative study, General Mental Health, University Learners

\section{Introduction}

Mental health refers to the successful performance of mental function, resulting in productive activities, fulfilling relationships with other people, and the ability to adapt to change and to cope with adversity. (Mental Health and Mental Disorders,2014)[1]. Mental health is indispensable for personal well-being, family and interpersonal relationships, and contribution to community or society. It is easy to overlook the value of mental health until problems surface. From early childhood to death, mental health is the catalyst of thinking and communication skills, learning emotional growth, resilience, and self-esteem. These are the ingredients of each individual's successful contribution to community and society where successful performance rests on a foundation of mental health (Satcher, February 2000 Vol. 31, No. 1) [2].

According to World Health Organization (WHO) the mental health is a complete state of physical, mental and social well being and not merely the absence of disease or infirmity". In 2002 WHO said that Mental health is the balance between all aspects of life- social, physical, spiritual and emotional. It impacts on how we manage our surroundings and make choices in our lives. It is an integral part of our overall health" (WHO, 2014) [3]. Recently WHO stresses that mental health "is not just the absence of mental disorder" [3].Mental health also refers to our cognitive and emotional wellbeing which tells about how we think, feel and behave (Nordqvist, 2015) [4]. WHO explains that especially in low and middle income countries, mental health services are very underfunded [4].

In fact the mental health and physical health are inseparable (Katz, Winter 1996, Volume 4, Issue 1) [5]. Mental functions related to somatic problem, anxiety and sleep disorder, social functioning and depression. Research reviewed for this report makes it clear that mental functions are carried out by a particular organ, the brain. Indeed, new and emerging technologies are making it increasingly possible for researchers to demonstrate the extent to which mental disorders and their treatment-both with medication and with psychotherapy-are reflected in physical changes in the brain (MentalHealth.Gov) [6].

Everyone experiences certain social and emotional difficulties in different stages of life. There might be issues from the past or concern about future, which is confusing and distressing the person and undermining the wellbeing. Any such issues can be managed with help, support and proper guidance. Therefore, by exploring those issues might show better path for teachers to educate their learners.

\section{Rationality}

It has been observed that many institutes prefer to discover general mental health of their people. Mental health is defined by the World Health Organization as a state of well-being in which every individual realizes his or her own potential, can cope with the normal stresses of life, can work productively and fruitfully, and is able to make a contribution to her or his community (MentalHealth.Gov) [6].Therefore, if we don't have a healthy mental state it will be hard for us to live our life to the fullest extent. Mental health disorders are common in many countries, and each year approximately one quarter of the population suffers from one or more mental disorders ( Mental Health and Mental Disorders, 2014)[1]. Maintaining a healthy emotional health can be a challenge for anyone. However, we can learn more about how our mental status can impact on us and discover coping mechanisms and find

\section{Volume 5 Issue 2, February 2016}




\section{International Journal of Science and Research (IJSR) \\ ISSN (Online): 2319-7064 \\ Index Copernicus Value (2013): 6.14 | Impact Factor (2014): 5.611}

helpful local and national resources. Positive mental health allows people to realize their full potential, cope with the stresses of life, work productively and make meaningful contributions to their communities (Johan Ormel, Michael VonKorff, T. Bedirhan Ustun, Stefano Pini, Ailsa Korten, \& Tineke Oldehinkel, December, 1994)[7]. The ways to maintain positive mental health include getting professional help if you need it, communicating with others, staying positive, getting physically active, helping others, getting enough sleep and developing coping skills (MentalHealth.Gov)[3]. For those reasons, identification of a person's mental health status is essential. Green university of Bangladesh has a unit named Psychosocial Counseling Unit (PSCU) which can easily discover mental health status of their students, teachers and staffs and take measures according to their needs. In June 2011, the Ontario government released the document Open Minds, Healthy Minds: Ontario's Comprehensive Mental Health and Addictions Strategy, which outlined a comprehensive strategy for addressing mental health and addiction problems of child and young learners (Supporting Minds, 2013)[8]. Mental health problems have a variety of causes and take a variety of forms. Their treatment may involve several types of intervention and support, which need to be delivered in an integrated and carefully coordinated way. The form of the response will be guided primarily by the insights and expertise of mental health professionals. However, because educators play an important role in the lives of most children and youth, they need to be aware of mental health issues that may affect students and understand how to contribute to a multifaceted response (Supporting Minds, 2013) [8].

\section{Research Questions}

The general objective of the study is to explore the mental health status of university learners and the research questions are-

1) What are the frequencies of social functioning, depression, somatic problem and anxiety \& sleep problem among the university learners?

2) What are the differences that exist according to their gender, semester, age and group of study on their mental health status?

3) What are the reasons behind any cases?

4) Is there any differences between public and private university learners' general mental health?

5) What are the suggestions to improve the general mental health of university learners?

\section{Literature Review}

In recent years the 12-item General Health Questionnaire (GHQ-12) has been extensively used as a short screening instrument, producing results that are comparable to longer versions of the GHQ (Michael H, Chris W, Paul R, Nigel J, Elizabenth M. \& Toby D., 2011) [9] The validity of the GHQ12 was compared with the GHQ-28 in a World Health Organization study of psychological disorders in general health care. Results are presented for 5438 patients interviewed in 15 centers using the primary care version of the Composite International Diagnostic Instrument or CIDI-PC. Results of the study were uniformly good, with the average area under the ROC curve 88 , range from 83 to 95 . Minor variations in the criteria used for defining a case made little difference to the validity of the GHQ, (P.Goldberg, et al., 1997) [10]. The GHQ was translated into 10 other languages for the purposes of this study, and validity coefficients were almost as high as in the original language. There was no tendency for the GHQ to work less efficiently in developing countries (Michael H, Chris W, Paul R, Nigel J, Elizabenth M., \& Toby D., 2011) [9]. Finally gender, age and educational level are shown to have no significant effect on the validity of the GHQ. If investigators wish to use a screening instrument as a case detector; the shorter GHQ is remarkably robust and works as well as the longer instrument. The latter should only be preferred if there is an interest in the scaled scores provided in addition to the total score (P.Goldberg, et al., 1997) [10].

A study was conducted on the psychometric properties of an existing measure of mental health by the GHQ-12 which revealed in three studies involving employees in an engineering firm $(n=659)$, recent school-leavers $(n=647)$, and unemployed men $(n=92)$. The measure was shown to be psychometrically sound in all cases, with a Likert scoring method providing a more acceptable distribution of scores. Scores on GHQ-12 were found to be much higher for those who were unemployed, higher for women than for men in one sample, and unrelated to age, job level and marital status (Michael H, Chris W, Paul R, Nigel J, Elizabenth M., \& Toby D., 2011) [9].To examine the impact of common mental illness on functional disability and the cross-cultural consistency for physical illness and to determine the level of disability associated with specific psychiatric disorders, a research was conducted on Common Mental Disorders and Disability across Cultures by WHO. Disability was most prominent among patients with major depression, panic disorder, generalized anxiety, and neurasthenia; disorderspecific differences were modest after controlling for psychiatric comorbidity. (Johan Ormel, Michael VonKorff, T. Bedirhan Ustun, Stefano Pini, Ailsa Korten, \& Tineke Oldehinkel, December, 1994) [7].

Meldrum et al (2009) note that mental disorders can affect a student's emotional wellbeing, ability to learn and can be a factor in why some students drop out of school (FroeseGermain \& Riel, 2012)[11].A study on the mental health of university students of United Kingdom assessed the levels of mental illness in undergraduate students. 1197 students were participated in this study. Rates of mental illness in students equaled those of the general population but only 5.1 percent were currently receiving treatment. Second year students reported the most significant increases in psychiatric symptoms (Macaskill, 2012) [12]. The Royal College of Psychiatrists (2011) provided a report to provide an update to previous document. The numbers of young people in higher education have expanded and they have become more socially and culturally diverse. Social changes such as withdrawal of

Volume 5 Issue 2, February 2016 


\section{International Journal of Science and Research (IJSR) \\ ISSN (Online): 2319-7064 \\ Index Copernicus Value (2013): 6.14 | Impact Factor (2014): 5.611}

financial support, higher rates of family breakdowns and economic recession are all having an impact on the well being of students (Mental health of students in higher education, September 2011) [13].

Doris Iarovici (2014) conducted a study on mental health issues and university students. The study found that students have lot of difficulties from adjustment disorder to mood disorder and a growing number of students are seeking help on campus. The study also found those students' lifestyle problems and psychiatric concerns using case vignettes to explore a variety of interventions. The problems of substance abuse, relationship difficulties, eating disorders, depression, anxiety and culture clashes are also included (Doris Iarovici, 2014)[14].

Therefore it is vital to explore university level learner's mental health status for ensuring their better academic and career development. Researches on this issue can contribute path and suggestions to develop curriculum to design teaching strategies to understand learners and to guide them in an adequate manners.

\section{Methodology}

This study has followed mixed approach of research. The population of the study was all the learners of public and private universities of Bangladesh. 303 students of private universities and 200 students from the public universities were randomly selected to participate for the survey (GHQ-28). A purposive sampling was used for selecting universities. Counseling sessions with students were considered as the source of qualitative data. Random sampling was used for selecting students from counseling sessions. Data gathering tools were General Health Questionnaire GHQ-28 and counseling sessions. Total sample sizes for survey and counseling session were 503 and 10 respectively. Multiinstrument analysis techniques based on statistical analysis were used in analyzing and interpreting collected raw data.

\section{Findings, Analysis \& Discussion}

Among 503 students, 303 were from private university and 200 were from public university. The students were taken for the research from different departments covering all the semesters and gender. The first objective of this study was to explore the general mental health status of public and private university learners. The table 1 and 2 show the status.

From the table 1, we can see that the frequency of somatic problem, anxiety \& sleep problem in severe cases was only near about $7 \%$ whereas students has less problem related to social functioning (3\%) and depression (2\%). It seems that students were in good mental health statuses. Table 2 illustrates that about $78 \%$ students were nonclinical cases and only $12 \%$ students were considered as clinical cases.
Table1: Frequencies of social functioning, depression, somatic and anxiety

\begin{tabular}{|c|c|c|c|c|}
\hline & $\begin{array}{c}\text { Somatic } \\
(\%)\end{array}$ & $\begin{array}{c}\text { Anxiety \& } \\
\text { Sleep Problem } \\
(\%)\end{array}$ & $\begin{array}{c}\text { Social Functioning } \\
(\text { SF })(\%)\end{array}$ & Depression \\
\hline Low & 50.3 & 57.5 & 76.9 & 89.5 \\
\hline Moderate & 37.2 & 34.6 & 18.3 & 4.4 \\
\hline Severe & 6.4 & 6.6 & 3.0 & 1.8 \\
\hline Total & 93.8 & 98.6 & 98.2 & 95.6 \\
\hline Missing & 6.2 & 1.4 & 1.8 & 4.4 \\
\hline
\end{tabular}

Table 2: Frequencies of Total General Mental Health (TGMH)

\begin{tabular}{|c|c|c|c|}
\hline \multicolumn{2}{|c|}{} & Frequency & Percent \\
\hline Valid & Non-clinical & 392 & 77.9 \\
\cline { 2 - 4 } & Clinical & 59 & 11.7 \\
\cline { 2 - 4 } & Total & 451 & 89.7 \\
\hline Missing & System & 52 & 10.3 \\
\hline \multicolumn{2}{|c|}{ Total } & 503 & 100.0 \\
\hline
\end{tabular}

However $10 \%$ data are missing related to this information. The reason behind it was students are not eager to answer the questions or they are hiding information. Moreover, the numbers of missing data were relatively high in all which was shown in the table 3 categories.

Table 3: Frequencies of Missing data

\begin{tabular}{|c|c|c|c|c|c|}
\hline & Somatic & Anxiety & Social & Depression & TMH \\
\hline Valid & 472 & 496 & 494 & 481 & 451 \\
\hline Missing & 31 & 7 & 9 & 22 & 52 \\
\hline
\end{tabular}

From counseling sessions it was found that students are not interested to share their personal issues due to lack of awareness and social stigma. From the figure1, it was found that general mental health of male learners was quite better than females in terms of somatic and anxiety \& sleep problem. Conversely, male students had more problems regarding social functioning than females in terms of severe cases.
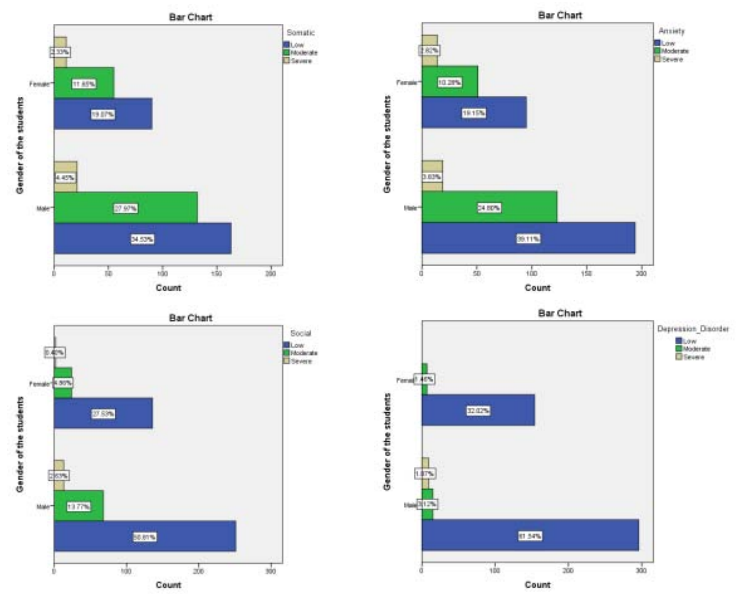

Figure 1: Gender wise Comparative TGMH

In the area of depression, the maximum students were quite sound. Very few male students were depressed in severe range where 


\section{International Journal of Science and Research (IJSR) \\ ISSN (Online): 2319-7064 \\ Index Copernicus Value (2013): 6.14 | Impact Factor (2014): 5.611}

as females were quite sound. From the counseling sessions girls were seemed more depressed than boys because of family issues, relationship problems and sexual abuses. And boys were more anxious than females. The reasons they mention for anxiety \& sleep problem were academic pressure, exam phobia, career concern, stress and family issues.
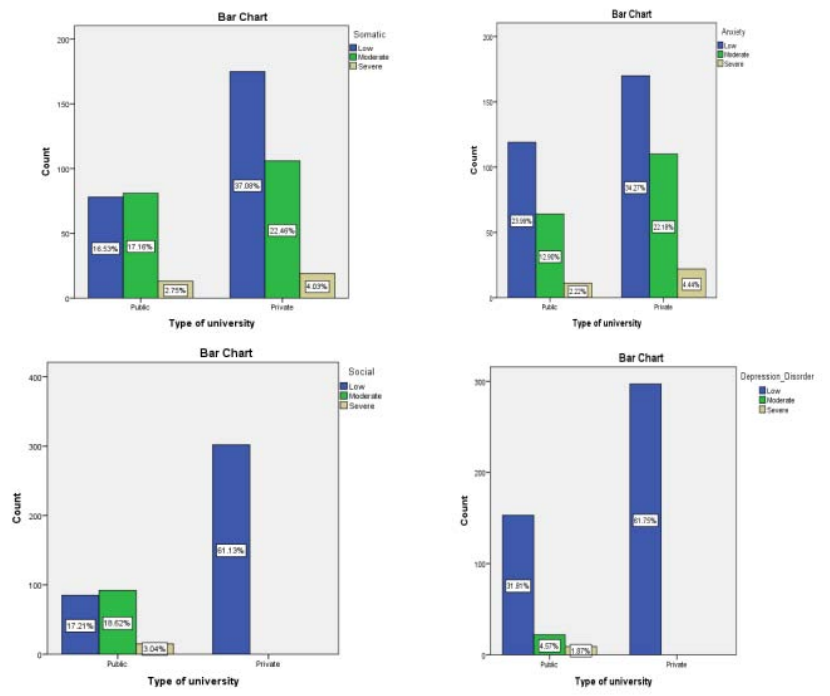

Figure 2: Type of University and Comparative TGMH
Private university learners had fewer problems in the areas of social functioning. Most of the public university learners were having depression because of session jam and choice of subject. Besides, maximum private university learners were having anxiety. The learners of this private university were getting the opportunities for taking counseling services from the counseling unit of this university. But in this public university there was no counseling unit, for this they were being deprived of getting mental health services from their university.

From the counseling sessions with the learners of this private university, some issues related to their mental health have been discovered like most of them were having relationship problems with their parents. Many of them were carrying high anxiety about their tuition fees, academic performance, career etc. All of them complained about their sleep problems. Besides some physical complaints were also present simultaneously that, loss of appetite, loss of weight, loss of interest in any activities, obsessions, feeling of sadness etc.

Maximum students have moderate and sound mental health between the ages of 20 years and 27 years. Afterwards, the tendency of sound mental health was decreasing due to career concern and academic pressure which were mentioned by the learners in the counseling session. Figure 3 has illustrated this finding.

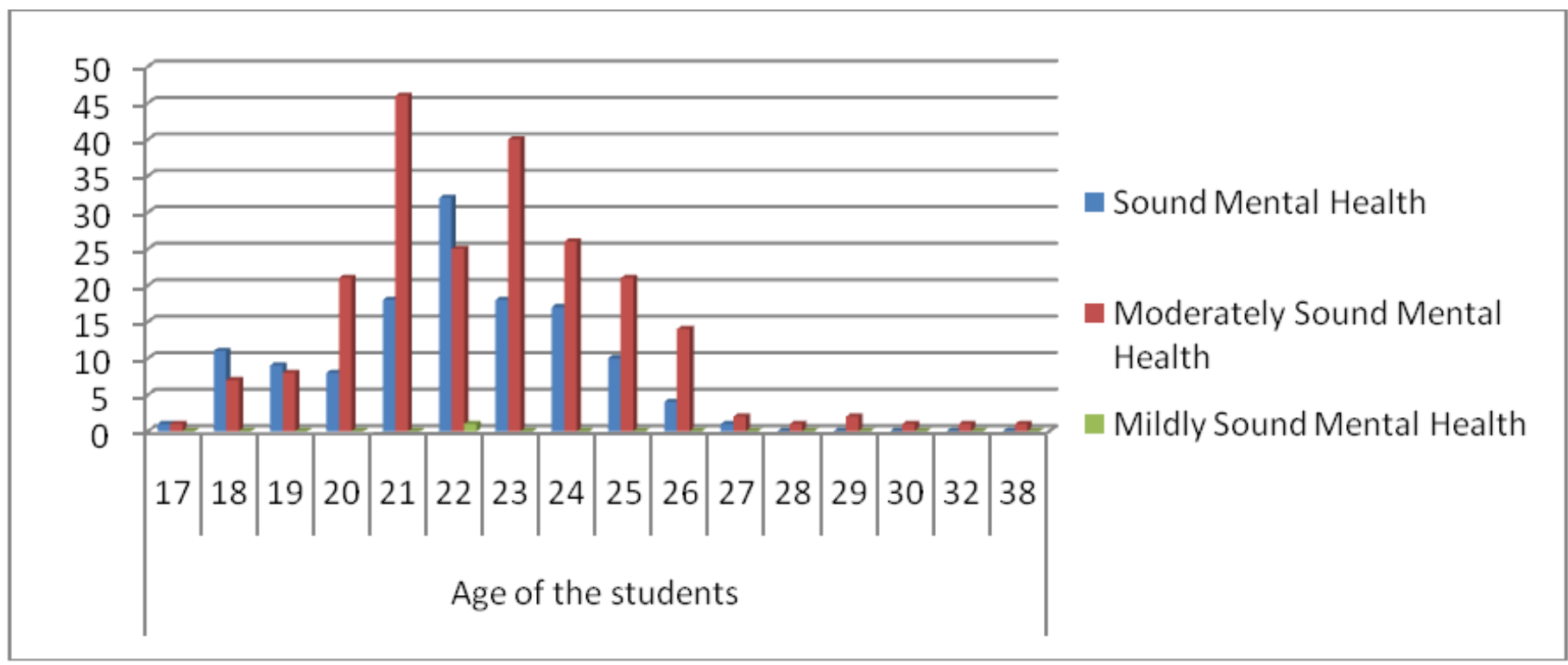

Figure 3: Total Mental Health Status related to Age

No significant association has been found between science and non science background students and their status of total mental health. 


\section{International Journal of Science and Research (IJSR)}

ISSN (Online): 2319-7064

Index Copernicus Value (2013): 6.14 | Impact Factor (2014): 5.611



Figure 4: Comparative Analysis

The fourth objective of this study was to investigate whether there is any differences exist between public and private university learners' general mental health. The research found a very little significant difference between the public and private university learners. Respectively about $.50 \%$ and $1 \%$ of public and private university learners were clinical cases, which mean public university learners' mental health is slightly sound than the private university learners. And mental health services are highly needed for this group. Among the public university learners about $10 \%$ and in the private university learners about $12 \%$ were nonclinical cases. This group may turn into the clinical cases in any time, for this reason necessary steps like awareness programs related to mental health issues, counseling services should be provided to maintain their sound mental health.

During the counseling sessions some suggestions came from the private university learners. They focused on providing workshops, awareness programs related to mental health issues. They shared that by these services university learners may learn the ways of maintaining their psychological wellbeing and counseling sessions are highly effective for their better academic performance and sharing their psychological problems or issues in such environment.

\section{Conclusion}

The findings of the study have showed that the mental health of the university learners is quite satisfactory but not totally free from psychological problems. A significant number of students are suffering from various somatic problems and anxiety \& sleep problems. And about $78 \%$ students were in nonclinical and only $12 \%$ students were in clinical level of general mental health. Somatic and Anxiety \& sleep problems were more in female learners than males. And males had more problems in social functioning than females in terms of severe cases. From the counseling sessions some issues related to their mental health were also discovered such as academic pressure, exam phobia, career concern, family problem, sexual abuse, obsessions, feeling of sadness etc. The present research reveals that there is a strong need of counseling services for ensuring the students' psychological wellbeing. And the psychosocial counseling unit may provide various psychological services and supports like workshops, training to enhance the students' awareness about the benefit of taking psychological services. These research findings may contribute to enhance their psychological wellbeing as well as their personal and professional wellbeing also. However, further research in this area is highly needed to explore other factors related to mental health.

\section{References}

[1] [1] Mental Health and Mental Disorders. (2014). Retrieved December 20, 2015, from Healthy People: http://www.healthypeople.gov/2020/topicsobjectives/topic/mental-health-and-mental-disorders

[2] [2] Satcher, M. (February 2000 Vol. 31, No. 1). Mental Health: A Report of the Surgeon General-Executive Summary. Professional Psychology: Research and Practice, 5-13.

[3] [3] WHO. (2014, August). Mental health: a state of wellbeing. Retrieved January 25, 2016, from World Health Organization http://www.who.int/features/factfiles/mental_health/en/

[4] [4] Nordqvist, C. (2015, November 3). What is mental health? What is mental disorder? Retrieved November 20, 2015, from Medical News Today (MNT): http://www.medicalnewstoday.com/articles/154543.php

[5] [5] Katz, I. R. ( Winter 1996, Volume 4, Issue 1). On the Inseparability of Mental and Physical Health in Aged Persons: Lessons From Depression and Medical Comorbidity. The American Journal of Geriatric Psychiatry, 1-16.

[6] [6] MentalHealth.Gov. (n.d.). Retrieved November 12, 2015, from U.S. Department of Health \& Human Services: http://www.mentalhealth.gov/basics/what-ismental-health/

[7] [7] Johan Ormel, P., Michael VonKorff, S., T. Bedirhan Ustun, M. P., Stefano Pini, M., Ailsa Korten, B., \& Tineke Oldehinkel, M. (December, 1994). Common Mental Disorders and Disability Across Cultures. The Journal of American Medical Association, 1741-1748.

[8] [8] Supporting Minds. (2013). Supporting Minds . Ontario , Canada: Ontario Public Service.

[9] [9] Michael H, B., Chris W, C., Paul R, J., Nigel J, K., Elizabenth M., S., \& Toby D., W. (2011). The use of the General Health Questionnaire as an indicator of mental health in occupational studies. Journal of Occupational Psychology, 187-194.

[10] [10] P.Goldberg, D., Gater, R., N.Sartoriys, B.Ustun, T., M.Piccinelli, Gureje, O., et al. (1997). The validity of two versions of the GHQ in the WHO study of mental illness in general health care. Psychological Medicine , 191-197.

[11][11] Froese-Germain, B., \& Riel, R. (2012). Understanding teachers' perspectives on student mental health. Ottawa: Canadian Teachers' Federation.

[12] [12] Macaskill, A. (2012). The mental health of university students in the United Kingdom. British Journal of Guidance and Counselling, 41 (4), 426-441.

[13][13](September 2011). Mental health of students in higher education. London : Royal College of Psychiatrists. 


\section{International Journal of Science and Research (IJSR) \\ ISSN (Online): 2319-7064 \\ Index Copernicus Value (2013): 6.14 | Impact Factor (2014): 5.611}

[14][14] Doris Iarovici, M. (2014, February ). Mental Health Issues and the University Student. Retrieved December 26, 2015, from Johns Hopkins University Press : https://jhupbooks.press.jhu.edu/content/mental-healthissues-and-university-student.

\section{Author Profile}

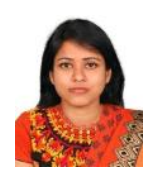

Arifa Rahman received her Master of Education from Institute of Education and Research, University of Dhaka, Bangladesh in 2010; the Bachelor of Education from the same institute in 2008. She is going to pursue her second master on special education at Flinders University with Endeavour Scholarship. Afterwards, she is going to pursue her Ph.D. degree in Social Media and Education from University of New South Wales. She was a teaching assistant, lecturer, lecturer II with BRAC Institute of Languages, BRAC University in 2010, 2011 and 2012 respectively. She has been working as Assistant Professor and lead facilitator at Green University Center of Excellence for Teaching and Learning, Green University of Bangladesh. Her research interests include teaching \& learning, technology based education, moral education and ethics teaching, social media and education, emotional intelligence, psychological issues and learning, special education and technology based education. At present, she is engaged in research on ethics teaching.

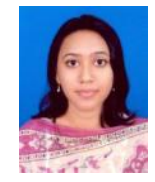

Shahrina Ferdous completed her M.Sc in Clinical Psychology from the University of Dhaka, Bangladesh in 2013 and the B.Sc in Psychology from the same university in 2010.She is going to pursue her M. Phil in Clinical Psychology at the University of Dhaka. She has been carrying an experience of four years in counseling services. She has been working as a Lecturer and Psychosocial Counselor at Green University Center of Excellence for Teaching and Learning, Green University of Bangladesh. Her research interests include adult mental health issues especially mental disorders, effect of psychotherapy, depressive disorder, physical illness and psychological wellbeing, teaching \& learning etc. 